\title{
On a class of singularly perturbed boundary value problems for which an adaptive mesh technique is necessary
}

\author{
P. W. Hemker and G. I. Shishkin ${ }^{1}$ \\ CWI, Amsterdam, The Netherlands, \\ IMM, Ural Branch of the Russian Academy of Science, Ekaterinburg, Russia.
}

\begin{abstract}
We construct discrete approximations for linear boundary value problems for elliptic differential equations for which the coefficient of the highest derivatives can take arbitrary small values from the interval $(0,1]$. Discretisation errors for classical discrete methods depend on the value of this parameter and can be of a size comparable with the solution of the original problem. We describe how to construct special discrete methods for which the accuracy of the discrete solution does not depend on the value of the parameter, and only depends on the number of mesh points used. We describe a class of boundary value problems, for which an adaptive mesh technique is necessary to obtain a parameter-uniform error-estimate as mentioned above. For this class of problems a special finite difference method is constructed. The solutions, obtained by the special method, converge in the discrete $l^{\infty}$-norm. For a model problem we show and compare results obtained by the classical method and those for the special scheme.
\end{abstract}

\section{Introduction}

For the solution of boundary value problems that have a smooth solution, well established methods can be used, such as finite difference, finite element or finite volume schemes (see e.g. [5]). The accuracy of the approximate solutions deteriorates when the solution becomes less smooth.

In the case of singularly perturbed differential equations, when the coefficient of the highest derivatives can take an arbitrary small value in $(0,1]$, the solution of the boundary value problems may have limited smoothness. The derivatives of such solutions may increase without bound when the small parameter tends to zero. When classical numerical methods are used, the error of the approximate solution depends on the value of the small parameter and may be of a size comparable with the solution of the original problem [4]. Therefore, for singularly perturbed boundary value problems it makes sense to construct special numerical methods, for which accuracy of approximate solution does not depend on the parameter, and for which the size of the error depends only on the number of nodal point used, i.e. methods which converge uniformly with respect to the small parameter (parameter-uniformly).

In $[2,4]$ special difference schemes were constructed for the solution of singularly perturbed boundary value problems, and it was proved that these schemes are parameter-

\footnotetext{
${ }^{1}$ This research was supported in part by the Dutch Research Organisation NWO through grant 07-30-012 .
} 
uniformly convergent. Those first strong results belong to two different approaches for the construction of special numerical methods for these problems with boundary layers:

(a) In the "fitted" method described in [4], the coefficients of difference equations are adapted such that a parameter-uniform accuracy of the approximate solution can be guaranteed on meshes with an arbitrary distribution of nodes (for example, on a uniform mesh);

(b) In the "adaptive mesh" method, described in [2], the usual classical discretisation scheme is used, but the nodes in the mesh are redistributed (condensed in the boundary layer) such that the parameter-uniform convergence is achieved.

The methods from the first approach are attractive because they allow the use of meshes with an arbitrary distribution of nodes, in particularly uniform grids (see, for example, $[1,4])$. Methods based on the second approach were constructed for a series of boundary value problems, e.g. see [8] (and references therein). For some boundary value problems, parameter-uniformly convergent schemes were constructed using both approaches for the same problem ([8]), or with the different approaches used to approximate the derivatives in different coordinate directions [7].

In this paper we consider a class of singularly perturbed boundary value problems for elliptic equations. Using the approaches (a) and (b), in Section 3 we introduce a natural class B of finite difference schemes for boundary value problems, in which we look for the special schemes which yield a parameter-uniformly convergent approximation to the solution.

The considered class of singularly perturbed convection-diffusion problems describe the diffusion of some material in a moving medium. For these boundary value problems we construct a special parameter-uniformly convergent schemes. We show that for schemes from class B the use of special adapted meshes is necessary. With a special adapted mesh we are able to construct finite difference schemes which converge parameter-uniformly. In Section 5 we give numerical results and compare the classical and the special finite difference schemes.

\section{The class of boundary value problems studied}

In many problems from physics we recognise the convective transport with diffusion described by

$$
-\varepsilon \Delta u(x)+<\vec{v}(x) \cdot \vec{\nabla}>u(x)=F(x), x \in \Omega .
$$

For the boundary of $\Omega$, which we assume to be piecewise smooth, we distinguish a wall, $\partial \Omega^{0}$, an inflow and an outflow boundary, $\partial \Omega^{+}$and $\partial \Omega^{-}$respectively. Clearly we have $\partial \Omega=\partial \Omega^{0} \cup \partial \Omega^{+} \cup \partial \Omega^{-}$and $\left\langle\vec{n}(x) \cdot \vec{v}(x)>=0, x \in \partial \Omega^{0} ;\left\langle\vec{n}(x) \cdot \vec{v}(x)><0, x \in \partial \Omega^{+}\right.\right.$; $<\vec{n}(x) \cdot \vec{v}(x)>>0, x \in \partial \Omega^{-}$. Here $\vec{n}(x)$ is the outward normal unit vector. At a wall, $\partial \Omega^{0}$, the boundary condition is written as

$$
\nu(u(x)-U(x))+\frac{\partial}{\partial n} u(x)=0, \quad x \in \partial \Omega^{0} .
$$

For $\nu \rightarrow \infty$ condition (2.1b) reduces to the Dirichlet condition. At $\partial \Omega^{+}$we assume the concentration to be known and at $\partial \Omega^{-}$we assume its diffusive component to be known:

$$
\begin{gathered}
u(x)=U^{+}(x), \quad x \in \partial \Omega^{+} \\
\frac{\partial}{\partial n} u(x)=\Psi(x), x \in \partial \Omega^{-} .
\end{gathered}
$$


When the parameter $\varepsilon$ tends to zero, we may expect a boundary layer to appear in the neighbourhood of the boundary $\partial \Omega^{0}$.

In this paper we describe a class of two-dimensional diffusive transport problems, for which we construct our special finite difference schemes. The problem is slightly more general than problem (2.1). On the rectangular domain $\Omega=\left\{x: 0<x_{i}<d_{i}, i=1,2\right\}$ we consider the elliptic boundary value problem for equation

$$
\begin{gathered}
L_{(2.2)} u(x) \equiv \quad\left\{\varepsilon^{2} \sum_{s=1,2} a_{s}(x) \frac{\partial^{2}}{\partial x_{s}^{2}}-b(x) \frac{\partial}{\partial x_{1}}-c(x)\right\} u(x)=f(x), \quad x \in \Omega, \\
u(x)=\varphi(x), \quad x \in \partial \Omega^{+}=\left\{x \mid x \in \partial \Omega, x_{1}=0\right\}, \\
l_{(2.2)} u(x) \equiv-\alpha \varepsilon \frac{\partial}{\partial n} u(x)-(1-\alpha) u(x)=\psi(x), \\
x \in \partial \Omega^{0}=\left\{x \mid x \in \partial \Omega, 0<x_{1}<d_{1}\right\}, \\
\frac{\partial}{\partial n} u(x)=\eta(x), x \in \partial \Omega^{-}=\left\{x \mid x \in \partial \Omega, x_{1}=d_{1}\right\} .
\end{gathered}
$$

Here $a_{s}(x), b(x), c(x), f(x), x \in \bar{\Omega} ; \varphi(x), x \in \partial \Omega^{+} ; \psi(x), x \in \partial \Omega^{0} ; \eta(x), x \in \partial \Omega^{-}$are sufficiently smooth and bounded functions, which satisfy

$$
0<a_{0} \leq a_{1}(x), a_{2}(x) \leq a^{0}, \quad 0<b_{0} \leq b(x), \quad c(x) \geq 0, x \in \bar{\Omega} .
$$

The parameters $\alpha$ and $\varepsilon$ may take any value from the intervals $\varepsilon \in(0,1], \alpha \in[0,1]$. When the parameter $\varepsilon$ tends to zero in $(2.2)$, in the neighbourhood of the boundary $\partial \Omega^{0}$ parabolic boundary layers may appear. We suppose that for the functions $\phi(x)$ and $\psi(x)$ compatibility conditions are satisfies to guarantee a continuous solution and derivative with respect to $x_{2}$ on the set $\partial \bar{\Omega}^{0} \cap \partial \Omega^{+}$.

The above class of problems includes, for example, the boundary value problem for the regular differential equation:

$$
L_{(2.3)} U(y) \equiv\left\{\sum_{s=1,2} A_{s}(y) \frac{\partial^{2}}{\partial y_{s}^{2}}-B(y) \frac{\partial}{\partial y_{1}}\right\} U(y)=F(y),
$$

for $y \in \widetilde{\Omega}$, with the regular boundary conditions

$$
\begin{array}{cc}
U(y)=\Phi(y), & y \in \tilde{\partial} \Omega^{+}, \\
l_{(2.3)} U(x) \equiv-\alpha \frac{\partial}{\partial n} U(y)-(1-\alpha) U(y)=\Psi(y), & y \in \tilde{\partial} \Omega^{0}, \\
\frac{\partial}{\partial n} U(y)=0, & y \in \tilde{\partial} \Omega^{-},
\end{array}
$$

on the rectangular domain $\tilde{\Omega}=\left\{y \mid 0<y_{i}<\tilde{d}_{i}, i=1,2\right\}, \tilde{d}_{i}=\varepsilon^{-1} d_{i}$.

As we shall see in the next section, for boundary value problem (2.2) classical finite difference approximations on a uniform grid [5] converge to the solution of (2.2) only for a fixed value of the parameter $\varepsilon$ (see Theorem 2.1). The accuracy of the numerical solution depends on this value of the parameter and it decreases with $\varepsilon$ until all accuracy is lost when the value of $\varepsilon / b_{0}$ is comparable with the mesh-size of the uniform grid. We shall show that classical finite difference schemes do not converge $\varepsilon$-uniformly (see Theorem 2.2 ) for small values of the parameter $\varepsilon$. Therefore it is our concern to construct special schemes which do converge $\varepsilon$-uniformly. In [7] a particular $\varepsilon$-uniformly convergent finite difference scheme was constructed for the special case of a Dirichlet problem for equation $(2.2 \mathrm{a})$. 
To solve of boundary value problem (2.2) we first study the classical finite difference method on a (possibly) non-uniform grid. On the set $\bar{\Omega}$ we introduce the rectangular grid

$$
\bar{\Omega}_{h}=\bar{\omega}_{1} \times \bar{\omega}_{2},
$$

where $\bar{\omega}_{s}$ is the (possibly) non-uniform grid on the interval $\left[0, d_{s}\right] ; N_{s}$ is the number of nodes in grid $\bar{\omega}_{s}, s=1,2$. Define $h_{s}^{i}=x_{s}^{i+1}-x_{s}^{i}, h_{s}=\max _{i} h_{s}^{i}, h=\max _{s} h_{s}$, $N=\min _{s} N_{s}, s=1,2, \Omega_{h}=\Omega \cap \bar{\Omega}_{h}, \partial \Omega_{h}=\partial \Omega \cap \bar{\Omega}_{h}$. For problem (2.2) we use difference scheme

$$
\begin{gathered}
\Lambda_{(2.5)} z(x)=f(x), x \in \Omega_{h}, \\
z(x)=\varphi(x), x \in \partial \Omega_{h}^{+}, \\
\lambda_{(2.5)} z(x)=\psi(x), x \in \partial \Omega_{h}^{0}, \\
\delta_{\overline{x_{1}}} z(x)=\eta(x), x \in \partial \Omega_{h}^{-},
\end{gathered}
$$

Here

$$
\begin{gathered}
\Lambda_{(2.5)} z(x) \equiv \varepsilon^{2} \sum_{s=1,2} a_{s}(x) \delta_{\overline{x_{s}} \widehat{x}_{s}} z(x)-b(x) \delta_{\overline{x_{1}}} z(x)-c(x) z(x), \\
\lambda_{(2.5)} z(x) \equiv\left\{\begin{array}{lll}
+\varepsilon \alpha \delta_{x_{2}} z(x) & -(1-\alpha) z(x), & x_{2}=0, \\
-\varepsilon \alpha \delta_{\overline{x_{2}}} z(x) & -(1-\alpha) z(x), & x_{2}=d_{2},
\end{array}\right.
\end{gathered}
$$

$\delta_{x_{s}} z(x)$ and $\delta_{\overline{x_{s}}} z(x)$ are the forward and backward difference and $\delta_{\overline{x_{s}} \widehat{x}_{s}} z(x)$ is the second difference on the non-uniform grid.

The difference scheme (2.4)-(2.5) is monotone (i.e. the maximum principle holds) [5].

By means of the maximum principle, and taking into account estimates of the derivatives, we find that the solution of the scheme (2.4)-(2.5) converges (for a fixed value of the parameter $\varepsilon$ ) as

$$
|u(x)-z(x)| \leq M \varepsilon^{-4} N^{-1}, \quad x \in \bar{\Omega}_{h} .
$$

Here and in the following we denote by $M$ (or $m$ ) a sufficiently large (or small) positive constants which do not depend on the value of parameter $\varepsilon$ or on the difference operators. The proof of (2.6) is similar to the classical proof of convergence for monotone difference schemes $[5,8]$. Taking into account an a-priori estimate for the solution this results in the following theorem.

Theorem 2.1 Let all $k$-th derivatives of the solution of boundary value problem (2.2) be bounded by $\mathcal{O}\left(\varepsilon^{-2 k}\right)$. Then, for a fixed value of parameter $\varepsilon$, the solution of the scheme (2.5)-(2.4) converges to solution of boundary value problem (2.2) with an error bound given by (2.6).

Clearly, from (2.6) no $\varepsilon$-uniform convergence of difference solution follows. In a report [3] we prove that it is impossible to get such an estimate for (2.4)-(2.5) on a fixed, $\varepsilon$ independent mesh. The proof follows the same lines as given in [6]: Thus we arrive at the following theorem.

Theorem 2.2 On a uniform grid the solution of the classical finite difference scheme (2.5) does not converge $\varepsilon$-uniformly to the solution of the boundary value problem (2.2). 


\section{Fitted difference scheme}

In this section we show that there does not exist a difference scheme, even based on a fitted method, for which the solution on a uniform grid converges $\varepsilon$-uniformly to the solution of boundary value problem (2.2). Let us consider the boundary value problem

$$
\begin{gathered}
L_{(3.7)} u(x) \equiv \varepsilon^{2} \Delta u(x)-\frac{\partial}{\partial x_{1}} u\left(x_{1}\right)=0, \quad x \in \Omega, \\
u(x)=0, \quad x \in \partial \Omega^{+}, \\
l_{(2.2)} u(x)=\psi(x), \quad x \in \partial \Omega^{0}, \\
\frac{\partial}{\partial n} u(x)=0, \quad x \in \partial \Omega^{-},
\end{gathered}
$$

where

$$
\psi(x)=\left\{\begin{array}{cll}
\psi_{0}\left(x_{1}\right), & x \in \partial \Omega^{0}, & x_{2}=0 \\
0, & x \in \partial \Omega^{0}, & x_{2} \neq 0
\end{array}\right.
$$

We assume that $\psi_{0}(0)=0$ and that $\psi_{0}\left(x_{1}\right), x_{1} \in\left[0, d_{1}\right]$ is sufficiently smooth.

For the solution of problem (3.7) a boundary layer appears near the wall for $x_{2}=0$. We know that the main term of asymptotic expansion of this solution is the solution of a parabolic boundary value problem, with solution $u_{\text {par }}$, and we have the estimate

$$
\left|u_{(3.7)}(x)-u_{\text {par }}(x)\right| \leq M \varepsilon, \quad x \in \bar{\Omega} .
$$

Now we introduce the class A of finite difference schemes for the model problem (3.7). It is defined by the use of a uniform mesh $\bar{\Omega}_{h}^{\text {unif }}$, and the usual, fitted five-point finite difference scheme for the approximation of equation (3.7a):

$$
\Lambda_{(3.8)} z(x) \equiv\left\{\sum_{s=1,2}\left(A_{s} \delta_{x_{s} \overline{x_{s}}}+B_{s} \delta_{x_{s}}-C\right\} z(x)=E . \quad x \in \Omega_{h} .\right.
$$

Here the coefficients $A_{s}, B_{s}, C, E$ are functionals derived from the coefficients of equation (3.7a), depending on $x, h_{1}, h_{2}$ and $\varepsilon$. We suppose that for $h_{2} \varepsilon^{-1} \rightarrow 0$ and $h_{1} \rightarrow 0$ the coefficients $A_{s}, B_{s}, C, E$ approximate (in $\ell^{\infty}$-norm) the coefficients in equation (3.7a), at least in the neighbourhood of a point near the boundary layer. We can prove (see [8]) the following statement.

Theorem 3.1 For the finite difference schemes in class A (i.e. on a uniform grid $\bar{\Omega}_{h}^{\text {unif }}$, there does not exist a discrete solution which converges $\varepsilon$-uniformly to the solution of boundary value problem (3.7).

A statement similar to Theorem 3.1 holds if we construct a difference scheme based on a stencil with an arbitrary, finite number of nodes. Theorem 3.1 and its generalisation can be understood as follows. All solutions of problem (3.7), for arbitrary functions $\psi(x)$ in (3.7e), are singular solutions. Even in the neighbourhood of the parabolic boundary layer, these solutions can not be represented as a linear combination of a finite set of functions of boundary layer type. Therefore we cannot choose a finite number of coefficients which define a finite difference operator such that it approximates the differential operator for the set of singular solutions. Thus, for a given ( $\varepsilon$-independent) grid we cannot expect to find a method that is $\varepsilon$-uniformly convergent for problem (2.2).

Let us now introduce the class B of finite difference schemes for problem (2.2). It is defined by the use of (non-uniform) rectangular grids $\bar{\Omega}_{h(2.4)}$ and, for the approximation 
of equation (2.2a), a (fitted) five-point finite difference stencil. The coefficients of the difference operator are functionals of the coefficients of equation (2.2a) and they depend on $x, \varepsilon$ and also on the mesh. We again suppose that for $h \rightarrow 0$ the coefficients of the difference operator approximate (in the $\ell^{\infty}$-norm) coefficients in equation $(2.2 \mathrm{a})$ on the set $\bar{\Omega}_{h(2.4)}$.

We remark that the class B is a natural class of finite difference schemes. This class includes methods both with fitted schemes and with fitted grids, i.e. it allows all freedom of choice mentioned in the introduction. The following statements can be derived from Theorem 3.1

Theorem 3.2 For the boundary value problem (2.2), there does not exist a finite difference scheme in class $\mathrm{B}$, based on a fitted scheme, for which the solution converges $\varepsilon$ -uniformly to solution of the boundary value problem (2.2) for a mesh with an arbitrary $\varepsilon$-independent distribution of nodes.

Theorem 3.3 For the boundary value problem (2.2), for schemes in class B special $\varepsilon$-dependent grids (adaptive meshes) are necessary for the construction of $\varepsilon$-uniformly convergent schemes.

Hence, we should conclude that, if we consider problems with parabolic boundary layers, within class B adaptive mesh techniques are necessary for the construction of $\varepsilon$-uniformly convergent difference schemes. Such an adaptive method should provide an (a-priori) $\varepsilon$ dependent mesh, or it should adapt its mesh during the computation, using either data from the problem (the given value of $\varepsilon$ ) or from the solution.

\section{The $\varepsilon$-uniformly convergent difference scheme}

By means of an adapted condensed mesh, in this section we construct an $\varepsilon$-uniformly convergent method for boundary value problem (2.2). From the previous section it is clear that we need a special mesh, condensed in the neighbourhood of boundary layers. How the nodes have to be placed is derived from a-priori estimates of the solution and its derivatives. Let us consider the special grid (see [8]),

$$
\bar{\Omega}_{h}^{*}=\bar{\omega}_{1} \times \bar{\omega}_{2}^{*},
$$

where $\bar{\omega}_{1}$ is a uniform mesh, and $\bar{\omega}_{2}^{*}=\bar{\omega}_{2}^{*}(\sigma)$ is a special piecewise uniform mesh; $\sigma$ is a parameter depending on $\varepsilon$ and $N_{2}$. The mesh $\bar{\omega}_{2}^{*}(\sigma)$ is constructed as follows. The interval $\left[0, d_{2}\right]$ is divided in three parts $[0, \sigma],\left[\sigma, d_{2}-\sigma\right]$ and $\left[d_{2}-\sigma, d_{2}\right], 0<\sigma \leq d_{2} / 4$. We divide each interval $[0, \sigma]$ and $\left[d_{2}-\sigma, d_{2}\right]$ in $N_{2} / 4$ equal parts and interval $\left[\sigma, d_{2}-\sigma\right]$ in $N_{2} / 2$ equal parts. We assume that $\sigma=\sigma\left(\varepsilon, N_{2}\right)=\min \left[d_{2} / 4, m \varepsilon \ln N_{2}\right]$, where $m=m_{(4.9)}$ is an arbitrary positive number.

Theorem 4.1 If the solution of (2.2) satisfies some estimates described in [3], then the solution of the discrete problem (2.5)-(4.9) converges $\varepsilon$-uniformly to the solution of boundary value problem (2.2). In particular, for the solution of the discrete problem the estimate holds:

$$
|u(x)-z(x)| \leq M N^{-1 / 3}, \quad x \in \bar{\Omega}_{h}^{*} .
$$

The proof of this theorem follows the lines of the proof in [8].

The difference scheme (2.5)-(4.9) belongs to class B. This scheme has typically an a-priori adapted mesh. 


\section{$5 \quad$ Numerical results}

By the theory (see Theorem 2.2) it is shown that the classical difference scheme (2.5) on the uniform grid does not converge in the $l^{\infty}$-norm $\varepsilon$-uniformly to the solution of the boundary value problem (2.2). But it would be possible that the error $\max _{\bar{\Omega}_{h}}|z(x)-u(x)|$ is not too large for the classical scheme. That would reduce the need for a special scheme.

On the other hand, Theorem 4.1 shows that the special scheme (2.5),(4.9) converges $\varepsilon$-uniformly, but no indication is given about the value of the order constant $M$ in (4.10) and the order of convergence is rather small. It might be possible that the error is rather large for any reasonable value of $N_{1}, N_{2}$. This might reduce the practical value of the special scheme. To decide on the practical value of the new scheme numerical experiments should give the final answer.

To see the effect of the special scheme in practice, we study the singularly perturbed elliptic equation with a mixed boundary condition.

$$
\begin{gathered}
L_{(5.11)} u(x) \equiv \varepsilon^{2} \Delta u(x)-\frac{\partial}{\partial x_{1}} u(x)=-1, \quad x \in \Omega, \\
l_{(5.11)} u(x)=\psi(x), \quad x \in \partial \Omega^{0}, \quad u(x)=0, \quad x \in \partial \Omega^{+}, \quad \frac{\partial}{\partial x_{2}} u(x)=0, \quad x \in \partial \Omega^{-},
\end{gathered}
$$

where

$$
l_{(5.11)} u(x) \equiv\left\{\begin{array}{rr}
\alpha \varepsilon \frac{\partial}{\partial x_{2}} u(x)-(1-\alpha) u(x), & x_{2}=0, \\
-\alpha \varepsilon \frac{\partial^{2}}{\partial x_{2}} u(x)-(1-\alpha) u(x), & x_{2}=1 .
\end{array}\right.
$$

We compare the numerical results for the scheme (2.5) on the uniform and on the special grid (4.9). Here $\Omega=\left\{x: 0<x_{1}, x_{2}<1\right\}$,

$$
\psi(x)= \begin{cases}x_{1}, & x \in \partial \Omega^{0}, x_{2}=0 \\ 0, & x \in \partial \Omega^{0}, x_{2}=1 .\end{cases}
$$

For the solution of problem (5.11) we have the representation

$$
u(x)=U(x)+W(x), \quad x \in \bar{\Omega},
$$

where $U(x)=x_{1}, x \in \bar{\Omega}$, is the outer solution, and $W(x)$ represents the parabolic boundary layer in the neighbourhood of the edges at $x_{2}=0$ and $x_{2}=1$. For the solution we have the estimate

$$
-1 \leq u(x) \leq 1, \quad x \in \bar{\Omega} .
$$

Due to Theorem 4.1 the solution of the discrete problem with the adapted mesh converges $\varepsilon$-uniformly to the solution of our model problem (5.11). The function $u_{h}(x)$, which is the solution of the special scheme (2.5)-(4.9) is shown in Figure 1.

To see the difference between the use of the uniform and the adapted grid, for the approximation of (5.11) we first use the uniform grid. We solve the problem for different values of the meshwidth $h_{1}=h_{2}=N^{-1}$ and for different values of the parameters $\varepsilon$ and $\alpha$. The results for a set of numerical experiments is given in Table 1. From Table 1 we can see that this solution does not converge $\varepsilon$-uniformly. For a fixed value of $N$, the errors depend on the parameters $\varepsilon$ and $\alpha$. For $\varepsilon \geq 0.1$ and $\alpha=0.0,0.1,0.5,1.0$ the error behaviour is regular: when $N$ increases, the error decreases. For $\varepsilon=10^{-3}$ and $\alpha=0.0,0.1,0.5$ and for $\varepsilon=10^{-2}$ and $\alpha=0.0$, for some values of $N$ the error increases with increasing $N$. For $\alpha=0.5,1.0$ and a fixed $N$ the error increases with decreasing $\varepsilon$. In particular, for $\varepsilon=10^{-3}$ and $\alpha=0.5,1.0$ the errors for $N \leq 128$ are of the same 
Figure 1. Solution computed with the adapted mesh: $\varepsilon=0.01, \alpha=0.5, N=32$ and $m=1.0$.

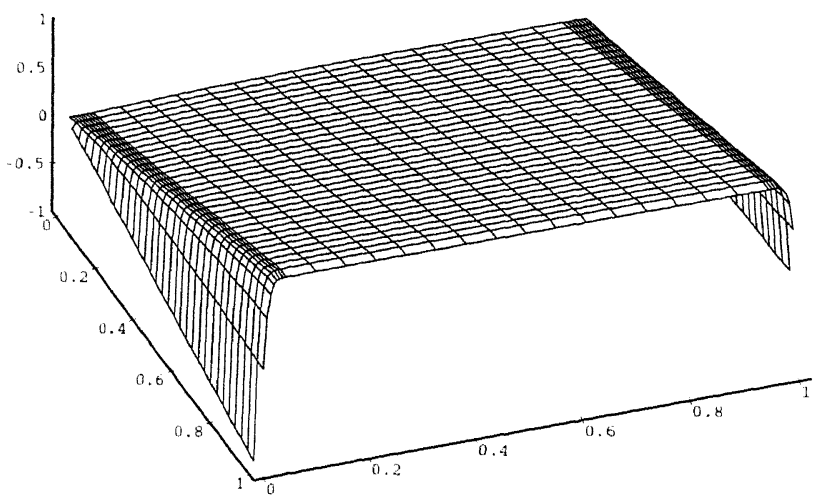

order or larger (in $\ell^{\infty}$-norm) than the solution of the BVP. Thus, the numerical results illustrate that the lack of $\varepsilon$-uniform convergence leads to large errors indeed.

We show the behaviour of $(2.5),(4.9)$, with $m=m_{(4.9)}=1$, applied to the model problem (5.11) The results for a set of numerical experiments is given in Table 2. We solve the problem for different values of $N, N=N_{1}=N_{2}$, and for different values of the parameters $\varepsilon$ and $\alpha$. From Table 2 we can see that the solution of the scheme (2.5)-(4.9) does converge $\varepsilon$-uniformly indeed. The errors for a fixed value of $\varepsilon=1.0,10^{-1}, 10^{-2}, 10^{-3}$ and $\alpha=0.0,0.1,0.5,1.0$ have all a regular behaviour and decrease for increasing $N$. For a fixed value of $\alpha$ and $N$ the error stabilises for decreasing $\varepsilon$ : the errors for $\varepsilon=10^{-2}$ and $\varepsilon=10^{-3}$ are practically the same. For $\varepsilon \leq 10^{-2}$ and a fixed value of $N$ we find the largest error for $\alpha=1.0$. In particular, for $\varepsilon \leq 10^{-2}, \alpha=1.0$ and $N=128$ the error is less than $6 \%$. Also here, the numerical results illustrate the practical value of $\varepsilon$-convergent methods.

\section{Conclusion}

For the elliptic boundary value problem (2.2), where a small parameter multiplies the highest derivative, we analysed different approaches for the construction of discrete methods. We studied methods for which the accuracy of the discrete solution does not depend on the value of the small parameter, but only on the number of points in the discretisation ( $\varepsilon$-uniform methods extended to non-uniform grids).

We show that in a natural class of finite difference schemes no $\varepsilon$-uniform methods exist on a uniform grid for the problem considered (Theorem 3.1). As a consequence, for the construction of $\varepsilon$-uniform methods the use of an adapted non-uniform mesh is necessary. With a special, adapted, non-uniform mesh and a simple classical difference scheme, we are able to construct an $\varepsilon$-uniform approximation

To illustrate the practical importance of our study, we show by a numerical example that, on a uniform grid, the classical difference scheme is inaccurate (and not $\varepsilon$-uniformly convergent). In our example, the error (with a Neumann boundary condition) is not less than $700 \%$ of the solution, for $N=128$ and $\varepsilon=10^{-3}$. The same example shows that we obtain an accurate, $\varepsilon$-uniformly convergent solution if we use the adapted mesh. Now 
Table 1. Table of errors $E(N, \varepsilon, \alpha)$ for the classical scheme.

In this table the error $E(N, \varepsilon, \alpha)$ is defined by

$$
\begin{gathered}
E(N, \varepsilon, \alpha)=\max _{x \in \bar{\Omega}_{h}}|e(x ; N, \varepsilon, \alpha)|, \\
e(x ; N, \varepsilon, \alpha)=z(x)-u^{\star}(x),
\end{gathered}
$$

where $u^{\star}(x)$ is the accurate interpolation of $z_{m}^{\star 256}(x), m=m_{(4.9)}=1$ (see Table 2), and $z(x) \equiv z^{N}(x)$ is the solution of (2.5), on the uniform grid with $h_{1}=h_{2}=N^{-1}$. Notice that $u^{\star}(x)$ is an accurate approximation of $u(x)$.

\begin{tabular}{|c|c|cccccc|}
\hline \multicolumn{2}{|c|}{$\mathrm{N}$} & 4 & 8 & 16 & 32 & 64 & 128 \\
\hline \multicolumn{1}{|c|}{} & $\alpha$ & & & & & & \\
\hline 1 & 0.00 & 0.105 & $0.633(-1)$ & $0.329(-1)$ & $0.158(-1)$ & $0.696(-2)$ & $0.241(-2)$ \\
$10^{-1}$ & & 0.305 & 0.144 & $0.655(-1)$ & $0.291(-1)$ & $0.122(-1)$ & $0.403(-2)$ \\
$10^{-2}$ & & 0.247 & 0.127 & $0.822(-1)$ & 0.107 & $0.882(-1)$ & $0.246(-1)$ \\
$10^{-3}$ & & 0.246 & 0.121 & $0.588(-1)$ & $0.283(-1)$ & $0.157(-1)$ & $0.200(-1)$ \\
\hline 1 & 0.10 & $0.691(-1)$ & $0.381(-1)$ & $0.170(-1)$ & $0.744(-2)$ & $0.314(-2)$ & $0.104(-2)$ \\
$10^{-1}$ & & 0.312 & 0.149 & $0.701(-1)$ & $0.310(-1)$ & $0.129(-1)$ & $0.423(-2)$ \\
$10^{-2}$ & & 0.247 & 0.194 & 0.185 & 0.156 & 0.109 & $0.583(-1)$ \\
$10^{-3}$ & & 0.246 & 0.209 & 0.216 & 0.216 & 0.211 & 0.197 \\
\hline 1 & 0.5 & $0.852(-1)$ & $0.401(-1)$ & $0.183(-1)$ & $0.842(-2)$ & $0.359(-2)$ & $0.119(-2)$ \\
$10^{-1}$ & & 0.706 & 0.473 & 0.254 & 0.121 & $0.526(-1)$ & $0.176(-1)$ \\
$10^{-2}$ & & 1.20 & 1.26 & 1.15 & 0.893 & 0.557 & 0.279 \\
$10^{-3}$ & & 1.28 & 1.43 & 1.49 & 1.48 & 1.41 & 1.27 \\
\hline 1 & 1.0 & 0.123 & $0.610(-1)$ & $0.288(-1)$ & $0.132(-1)$ & $0.562(-2)$ & $0.187(-2)$ \\
$10^{-1}$ & & 1.52 & 0.752 & 0.344 & 0.154 & $0.643(-1)$ & $0.211(-1)$ \\
$10^{-2}$ & & 18.2 & 10.3 & 5.19 & 2.40 & 1.02 & 0.408 \\
$10^{-3}$ & & 187. & 109. & 57.9 & 29.5 & 14.6 & 7.01 \\
\hline
\end{tabular}

the error is not larger than $6 \%$ of the solution, for any value of the parameter $\varepsilon$. Thus, the numerical example illustrates that the theoretical considerations have significant practical implications.

\section{References}

[1] D.N. Allen and R.V. Southwell. Relaxation method applied to determine the motion, in two dimensions, of viscous fluid past a fixed cylinder. Quart. J. Mech. Appl. Math., 8:129-145, 1955.

[2] N.S. Bakhvalov. On optimization of methods to solve boundary value problems with boundary layers. Zh. Vychisl. Mat. Mat. Fiz., 9:841-859, 1969. (in Russian).

[3] P. W. Hemker and G. I. Shishkin. Singularly perturbed boundary value problems for which an adaptive mesh technique is necessary. 1993, to appear.

[4] A.M. Il'in. Difference scheme for a differential equation with a small parameter at the highest-order. Mat. Zametki, 6:237-248, 1969. (in Russian).

[5] A.A. Samarsky. Theory of Difference Scheme. Nauka, Moscow, 1989. (in Russian). 
Table 2. Table of errors $E(N, \varepsilon, \alpha)$ for the special scheme.

In this table the function $E(N, \varepsilon, \alpha)$ is defined by (5.12), but now $z(x)=z_{m}^{\star N}(x)$ in (5.12) is the solution of $(2.5),(4.9)$ with $m=m_{(4.9)}=1$.

\begin{tabular}{|c|c|cccccc|}
\hline \multicolumn{2}{|c|}{$\mathrm{N}$} & 4 & 8 & 16 & 32 & 64 & 128 \\
\hline$\varepsilon$ & $\alpha$ & & & & & & \\
\hline 1 & 0.0 & 0.105 & $0.633(-1)$ & $0.329(-1)$ & $0.158(-1)$ & $0.696(-2)$ & $0.241(-2)$ \\
$10^{-1}$ & & 0.262 & 0.144 & $0.655(-1)$ & $0.291(-1)$ & $0.122(-1)$ & $0.403(-2)$ \\
$10^{-2}$ & & 0.246 & 0.147 & $0.807(-1)$ & $0.361(-1)$ & $0.148(-1)$ & $0.497(-2)$ \\
$10^{-3}$ & & 0.246 & 0.147 & $0.807(-1)$ & $0.361(-1)$ & $0.148(-1)$ & $0.497(-2)$ \\
\hline 1 & 0.1 & $0.691(-1)$ & $0.381(-1)$ & $0.170(-1)$ & $0.744(-2)$ & $0.314(-2)$ & $0.104(-2)$ \\
$10^{-1}$ & & 0.276 & 0.149 & $0.701(-1)$ & $0.310(-1)$ & $0.129(-1)$ & $0.423(-2)$ \\
$10^{-2}$ & & 0.246 & 0.170 & $0.887(-1)$ & $0.461(-1)$ & $0.240(-1)$ & $0.924(-2)$ \\
$10^{-3}$ & & 0.246 & 0.169 & $0.887(-1)$ & $0.461(-1)$ & $0.241(-1)$ & $0.925(-2)$ \\
\hline 1 & 0.5 & $0.852(-1)$ & $0.401(-1)$ & $0.183(-1)$ & $0.842(-2)$ & $0.359(-2)$ & $0.119(-2)$ \\
$10^{-1}$ & & 0.611 & 0.473 & 0.254 & 0.121 & $0.526(-1)$ & $0.176(-1)$ \\
$10^{-2}$ & & 0.539 & 0.511 & 0.361 & 0.217 & 0.111 & $0.420(-1)$ \\
$10^{-3}$ & & 0.535 & 0.511 & 0.361 & 0.217 & 0.111 & $0.420(-1)$ \\
\hline 1 & 1.0 & 0.123 & $0.610(-1)$ & $0.288(-1)$ & $0.132(-1)$ & $0.562(-2)$ & $0.187(-2)$ \\
$10^{-1}$ & & 1.14 & 0.752 & 0.344 & 0.154 & $0.643(-1)$ & $0.211(-1)$ \\
$10^{-2}$ & & 0.977 & 0.889 & 0.554 & 0.301 & 0.144 & $0.521(-1)$ \\
$10^{-3}$ & & 0.963 & 0.888 & 0.554 & 0.301 & 0.144 & $0.521(-1)$ \\
\hline
\end{tabular}

[6] G.I. Shishkin. An increase in accuracy of solutions to difference scheme for parabolic: equations with small parameters at highest-order derivatives. Zh. Vychisl. Mat. Mat. Fiz., 24:864 875, 1984. (in Russian).

[7] G.I. Shishkin. Approximation of solutions to singularly perturbed boundary value problems with corner boundary layers. Zh. Vychisl. Mat. Mat. Fiz., 27:1360 1374, 1987. (in Russian).

[8] G.I. Shishkin. Approximation of solutions to singularly perturbed boundary value problems with parabolic boundary layers. Zh. Vychisl. Mat. Mat. Fiz., 29:963 977, 1989. (in Russian). 